\title{
On the Computation of a Bivariate $t$-Distribution*
}

\author{
By D. E. Amos and W. G. Bulgren
}

Abstract. The cumulative bivariate $t$-distribution associated with random variables $T_{1}=X_{1} /(S / k)^{1 / 2}, T_{2}=X_{2} /(S / k)^{1 / 2}$ is considered where $X_{1}, X_{2}$ are bivariate normal with correlation coefficient $\rho$ and $S$ is an independent $\chi^{2}$ random variable with $k$ degrees of freedom. Representations in terms of series and simple, one-dimensional quadratures are presented together with efficient computational procedures for the special functions used in numerical evaluation.

Preliminary Representations. The bivariate $t$-distribution derived below has been of interest to many authors [1], [3], [8], [15]. The work of Dunnett and Sobel [2] on the cumulative distribution in terms of incomplete beta functions stands out for computational convenience. These results, coupled with the more recent work of Gautschi [7] on efficient computational procedures for many of the special functions, makes these results even more accessible. The need for other computational formulae stems from possible losses of significance by subtraction in numerical evaluation. A simple quadrature derived below overcomes this difficulty and certain series representations offer computational advantages for large degrees of freedom.

The usual procedure for deriving this $t$-distribution starts with the bivariate normal with correlation matrix $\Sigma$ associated with the random variables $X_{1}, X_{2}$ and a $\chi^{2}$-distribution with $k$ degrees of freedom associated with an independent random variable $S$,

$$
n\left(x_{1}, x_{2}\right)=\frac{1}{2 \pi|\Sigma|^{1 / 2}} \exp \left[-\left(\hat{x} \cdot \Sigma^{-1} \hat{x}\right) / 2\right], \quad f(s)=\frac{1}{2^{k / 2} \Gamma(k / 2)} s^{-1+k / 2} e^{-s / 2} .
$$

The distribution of the variables $T_{1}=X_{1} /(S / k)^{1 / 2}$ and $T_{2}=X_{2} /(S / k)^{1 / 2}$ is constructed according to

$$
P\left(T_{1} \leqq t_{1}, T_{2} \leqq t_{2}\right)=\int_{0}^{\infty} N\left(X_{1} \leqq t_{1}\left(\frac{s}{k}\right)^{1 / 2}, X_{2} \leqq t_{2}\left(\frac{s}{k}\right)^{1 / 2} \mid s\right) f(s) d s
$$

where $N$ is the cumulative of the distribution $n\left(x_{1}, x_{2}\right)$.

An exchange of integrals and a scaling of the variables yields

$$
\begin{aligned}
P\left(T_{1} \leqq t_{1}, T_{2} \leqq t_{2}\right)= & \frac{1}{2 \pi|\Sigma|^{1 / 2} \Gamma(1+k / 2)} \\
& \times \int_{-\infty}^{t_{2}} \int_{-\infty}^{t_{1}} \int_{0}^{\infty} \exp \left[-u\left[1+Q\left(v_{1}, v_{2}\right) / k\right]\right] u^{k / 2} d u d v_{1} d v_{2} \\
= & \frac{1}{2 \pi|\Sigma|^{1 / 2}} \int_{-\infty}^{t_{2}} \int_{-\infty}^{t_{1}} \frac{d v_{1} d v_{2}}{\left[1+\frac{1}{k} Q\left(v_{1}, v_{2}\right)\right]^{1+k / 2}} .
\end{aligned}
$$

Received April 22, 1968.

* This work was supported in part by the United States Atomic Energy Commission. 
Here the integrand is the density function for $T_{1}$ and $T_{2}$ with

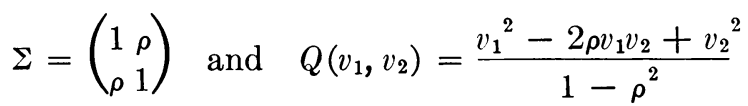

where $\rho,-1<\rho<1$, is the correlation coefficient. The exchanges of integrals and sums are justified on the basis of absolute convergence.

Quadrature Formulae. We start with (1) in the form

$$
P=\frac{1}{\pi|\Sigma|^{1 / 2} \Gamma(k / 2)} \int_{0}^{\infty} u^{k / 2} e^{-u} \int_{-\infty}^{t_{2} / N k} \int_{-\infty}^{t_{1} / N k} \exp \left[-u Q\left(x_{1}, x_{2}\right)\right] d x_{1} d x_{2} d u
$$

and rotate the $x_{1}, x_{2}$ axes so that the quadratic form

$$
Q\left(x_{1}, x_{2}\right)=\frac{x_{1}^{2}-2 \rho x_{1} x_{2}+x_{2}^{2}}{1-\rho^{2}}=\left(\hat{x} \cdot \Sigma^{-1} \hat{x}\right)
$$

has only sums of squares. The eigenvalues of $\Sigma^{-1}$ are $\lambda_{1,2}=1 /(1 \pm \rho)$ with eigenvectors the columns of

$$
L=\left(\begin{array}{cc}
\frac{1}{2^{1 / 2}} & \frac{-1}{2^{1 / 2}} \\
\frac{1}{2^{1 / 2}} & \frac{1}{2^{1 / 2}}
\end{array}\right)
$$

This $45^{\circ}$ rotation of axes $\hat{x}=L \hat{v}$ reduces $Q$ to $\lambda_{1} v_{1}^{2}+\lambda_{2} v_{2}{ }^{2}$. A further change of scale $\hat{v}=M \hat{w}$ with

$$
M=\left(\begin{array}{cc}
\frac{1}{\lambda_{1}^{1 / 2}} & 0 \\
0 & \frac{1}{\lambda_{2}^{1 / 2}}
\end{array}\right)
$$

gives $Q=w_{1}^{2}+w_{2}^{2}$ under the transformation $\hat{x}=L M \hat{w}$. The region of integration in the $w_{1}, w_{2}$ plane is now the sector labeled $R_{w}$ in Fig. 1. The determinant $|L M|=|\Sigma|^{1 / 2}$ is the Jacobian of the transformation.
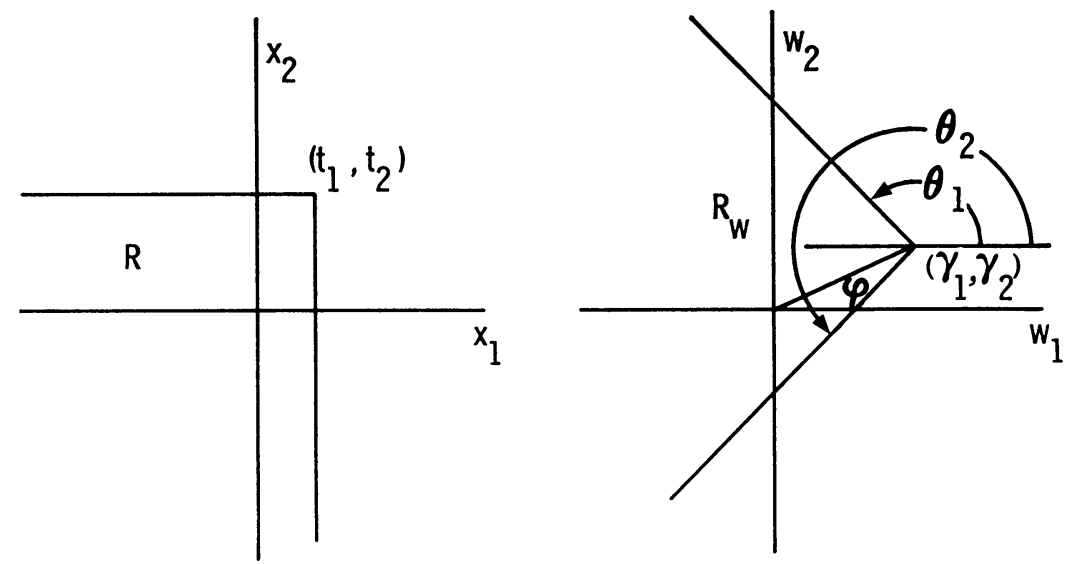

Figure 1 
The point $\left(t_{1} / k^{1 / 2}, t_{2} / k^{1 / 2}\right)$ transforms into the point $\left(\gamma_{1}, \gamma_{2}\right)$ where

$$
\begin{array}{rlrl}
\gamma_{1} & =\left(t_{2}+t_{1}\right)\left(\frac{\lambda_{1}}{2 k}\right)^{1 / 2}, & \gamma_{2}=\left(t_{2}-t_{1}\right)\left(\frac{\lambda_{2}}{2 k}\right)^{1 / 2} \\
\theta_{1} & =\pi-\tan ^{-1}\left(\frac{1+\rho}{1-\rho}\right)^{1 / 2}, \quad \theta_{2}=\pi+\tan ^{-1}\left(\frac{1+\rho}{1-\rho}\right)^{1 / 2} \\
\varphi & =\tan ^{-1} \frac{\gamma_{2}}{\gamma_{1}}, \quad \gamma_{1}>0 \\
& =\pi+\tan ^{-1} \frac{\gamma_{2}}{\gamma_{1}}, \quad \gamma_{1}<0 .
\end{array}
$$

Here we take $-\pi / 2<\tan ^{-1} z<\pi / 2$ with the usual convention $\varphi=\pi / 2$ for $\gamma_{1}=0$ and $\gamma_{2}>0, \varphi=-\pi / 2$ for $\gamma_{1}=0$ and $\gamma_{2}<0$ and $\varphi \equiv 0$ for $\gamma_{1}=\gamma_{2}=0$. The integration now proceeds in polar coordinates

$$
\begin{aligned}
P= & \frac{1}{\pi \Gamma(k / 2)} \int_{0}^{\infty} u^{k / 2} e^{-u} \int_{\theta_{1}}^{\theta_{2}} \int_{0}^{\infty} r \\
& \times \exp \left(-u\left[\left(\gamma_{1}+r \cos \theta\right)^{2}+\left(\gamma_{2}+r \sin \theta\right)^{2}\right]\right) d r d \theta d u
\end{aligned}
$$

$$
\begin{aligned}
P= & \frac{1}{2 \pi \Gamma(k / 2)} \int_{\theta_{1}}^{\theta_{2}} d \theta \int_{0}^{\infty} u^{-1+k / 2} \\
& \times \exp \left(-\left(1+\gamma_{1}{ }^{2}+\gamma_{2}{ }^{2}\right) u\right) \int_{0}^{\infty} \exp \left(-v-2(u v)^{1 / 2} K(\theta)\right) d v d u
\end{aligned}
$$

after a change of variables $r=(v / u)^{1 / 2}$. The last integral is related to the parabolic cylinder function, $D_{-2}$, and

$$
P=\frac{1}{2 \pi \Gamma(k / 2)} \int_{\theta_{1}}^{\theta_{2}} \int_{0}^{\infty} u^{-1+k / 2} e^{-I(\theta) u} D_{-2}\left[(2 u)^{1 / 2} K(\theta)\right] d u d \theta
$$

where

$$
\begin{aligned}
& H(\theta)=1+{\gamma_{1}}^{2}+{\gamma_{2}}^{2}-\frac{K^{2}(\theta)}{2}, \\
& K(\theta)=\gamma_{1} \cos \theta+\gamma_{2} \sin \theta=\left({\gamma_{1}}^{2}+{\gamma_{2}}^{2}\right)^{1 / 2} \cos (\theta-\varphi) .
\end{aligned}
$$

Case $1 . K(\theta) \geqq 0$ or $\cos (\theta-\varphi) \geqq 0$.

In this case the Laplace transform is readily available from tables (see formula (15) of the Appendix) giving

$$
P_{1}=\frac{1}{2 \pi(k+1)\left(1+\gamma_{1}{ }^{2}+\gamma_{2}{ }^{2}\right)^{k / 2}} \int_{\theta_{1}}^{\theta_{2}} F\left(1, \frac{k}{2} ; \frac{k+3}{2} ; 1-c^{2} \cos ^{2}(\theta-\varphi)\right) d \theta
$$

where $c=\left(\left(\gamma_{1}^{2}+\gamma_{2}^{2}\right) /\left(1+\gamma_{1}^{2}+\gamma_{2}^{2}\right)\right)^{1 / 2}$ and $F$ is the Gauss hypergeometric function. For numerical evaluation, the right side of the expression

$$
1-c^{2} \cos ^{2}(\theta-\varphi)=\frac{1+\left({\gamma_{1}}^{2}+{\gamma_{2}}^{2}\right) \sin ^{2}(\theta-\varphi)}{1+\gamma_{1}{ }^{2}+{\gamma_{2}}^{2}}
$$

is used to prevent losses of significance when $c \cos (\theta-\varphi)$ is close to 1 . The series for the hypergeometric function is fairly rapidly convergent with all positive terms, 
although the analytic continuation formula is valuable when $\sin (\theta-\varphi)$ is close to 1 [4, p. 108]:

$$
\begin{aligned}
F\left(1, \frac{k}{2} ; \frac{k+3}{2} ; 1-x\right)= & (k+1) F\left(1, \frac{k}{2} ; \frac{1}{2} ; x\right) \\
& -\frac{2 \pi^{1 / 2} \Gamma((k+3) / 2)}{\Gamma(k / 2)} \sqrt{ } x(1-x)^{-(k+1) / 2}, \quad x \geqq 0 .
\end{aligned}
$$

The parameters of this $F$ function also satisfy a condition for quadratic transformations of the argument [4, p. 64]. Exchanging the order of integration in (3) leads to formula (5) through $D_{-k}$. There is an alternate form which corresponds with results of Case 2 which is obtained in the Appendix, namely

$$
\begin{aligned}
& P_{1}=\frac{1}{2 \pi(k+1)\left(1+\gamma_{1}{ }^{2}+\gamma_{2}{ }^{2}\right)^{k / 2}} \\
& \times \int_{\theta_{1}}^{\theta_{2}} \frac{F\left(\frac{k-1}{2}, \frac{3-k}{2} ; \frac{k+3}{2} ; \frac{1-c|\cos (\theta-\varphi)|}{2}\right)}{\left[\frac{1+c|\cos (\theta-\varphi)|}{2}\right]^{(k+1) / 2}} d \theta .
\end{aligned}
$$

Case 2. $K(\theta)<0$ or $\cos (\theta-\varphi)<0$.

In this case the results for the Laplace transform (4) are presented in the Appendix. The application of (16) in (4) gives

$$
\begin{aligned}
& P_{2}=\frac{1}{2 \pi(k+1)\left(1+\gamma_{1}{ }^{2}+\gamma_{2}{ }^{2}\right)^{k / 2}} \\
& \times \int_{\theta_{1}}^{\theta_{2}} \frac{F\left(\frac{k-1}{2}, \frac{3-k}{2} ; \frac{k+3}{2} ; \frac{1+c|\cos (\theta-\varphi)|}{2}\right)}{\left[\frac{1-c|\cos (\theta-\varphi)|}{2}\right]^{(k+1) / 2}} d \theta .
\end{aligned}
$$

The series for the integrand in numerical evaluation is not useful for large $k$ because of losses of significance due to small differences of large numbers, although the ultimate convergence rate is faster than in Case 1. For odd $k$ in (7) and (8) the $F$ function is a polynomial of degree $\max \{0,(k-3) / 2\}$ which is best computed by the analytic continuation formula

$$
\begin{aligned}
F\left(\frac{k-1}{\iota}, \frac{3-k}{2} ; \frac{k+3}{2} ; x\right)= & \frac{\Gamma\left(\frac{k+3}{2}\right) \Gamma\left(\frac{k+1}{2}\right)}{\Gamma(k)} F\left(\frac{k-1}{2}, \frac{3-k}{2} ; \frac{1-k}{2} ; 1-x\right) \\
& +\epsilon_{k}(1-x)^{(k+1) / 2} F\left(2, k ; \frac{k+3}{2} ; 1-x\right)
\end{aligned}
$$

where

$$
\begin{aligned}
\epsilon_{k} & =0, & k \text { odd } \\
& =1, & k \text { even }
\end{aligned}
$$

since most terms of the $F$ on the right are positive. For even $k$, neither series on the 
right terminates and the convergence is slow for $x$ close to zero and $k$ large. Thus, for $k$ large and even and $x$ near the middle of $[0,1]$, one can expect poor results in numerical computation.

The defects of this case can be remedied however by considering

$$
D_{-\nu}(z)=D_{-\nu}(-z)+2^{(1-\nu) / 2} \frac{\Gamma(-1 / 2)}{\Gamma(\nu / 2)} z e^{-z^{2} / 4} \Phi\left(\frac{1+\nu}{2}, \frac{3}{2} ;-\frac{z^{2}}{2}\right)
$$

in Eq. (4) where $\Phi$ is the confluent hypergeometric function. Then we have

$$
P_{2}=P_{1}-\frac{\Gamma\left(\frac{k+1}{2}\right)}{\sqrt{ } \pi \Gamma\left(\frac{k}{2}\right)\left(1+\gamma_{1}{ }^{2}+\gamma_{2}{ }^{2}\right)^{k / 2}} \int_{\theta_{1}}^{\theta_{2}} \frac{c \cos (\theta-\varphi) d \theta}{\left[1-c^{2} \cos ^{2}(\theta-\varphi)\right]^{(k+1) / 2}}
$$

since $\Phi(3 / 2,3 / 2 ; x)=e^{x}$.

In summary, the quadrature formulae may be expressed by (i)

$$
P=\frac{1}{2 \pi(k+1)\left(1+\gamma_{1}{ }^{2}+\gamma_{2}{ }^{2}\right)^{k / 2}} \int_{\theta_{1}}^{\theta_{2}} I(\theta) d \theta
$$

where $I(\theta)$ may have one of the forms,

$$
I(\theta)=\frac{F\left(\frac{k-1}{2}, \frac{3-k}{2} ; \frac{k+3}{2} ; \frac{1-c \cos (\theta-\varphi)}{2}\right)}{\left[\frac{1+c \cos (\theta-\varphi)}{2}\right]^{(k+1) / 2}}
$$

or

$$
\begin{aligned}
I(\theta) & =F\left(1, \frac{k}{2} ; \frac{k+3}{2} ; \frac{1+\left(\gamma_{1}{ }^{2}+\gamma_{2}{ }^{2}\right) \sin ^{2}(\theta-\varphi)}{1+\gamma_{1}{ }^{2}+\gamma_{2}{ }^{2}}\right), \quad \cos (\theta-\varphi) \geqq 0 \\
& =\frac{F\left(\frac{k-1}{2}, \frac{3-k}{2} ; \frac{k+3}{2} ; \frac{1+c|\cos (\theta-\varphi)|}{2}\right)}{\left[\frac{1-c|\cos (\theta-\varphi)|}{2}\right]^{(k+1) / 2}}, \quad \cos (\theta-\varphi)<0
\end{aligned}
$$

and (ii)

$$
\begin{aligned}
P= & \frac{1}{2 \pi(k+1)\left(1+\gamma_{1}{ }^{2}+\gamma_{2}^{2}\right)^{k / 2}} \int_{\theta_{1}}^{\theta_{2}} F\left(1, \frac{k}{2} ; \frac{k+3}{2} ; 1-c^{2} \cos ^{2}(\theta-\varphi)\right) d \theta \\
& -\frac{\Gamma\left(\frac{k+1}{2}\right)}{\sqrt{ } \pi \Gamma\left(\frac{k}{2}\right)\left(1+\gamma_{1}{ }^{2}+\gamma_{2}{ }^{2}\right)^{k / 2}} \int_{\theta_{1}}^{\theta_{2}} \frac{\delta(\theta) c \cos (\theta-\varphi)}{\left[1-c^{2} \cos ^{2}(\theta-\varphi)\right]^{(k+1) / 2}} d \theta
\end{aligned}
$$

where

$$
\begin{aligned}
\delta(\theta) & =0 \quad \text { if } \cos (\theta-\varphi) \geqq 0 \\
& =1 \quad \text { if } \cos (\theta-\varphi)<0
\end{aligned}, \quad c=\left(\frac{\gamma_{1}{ }^{2}+\gamma_{2}{ }^{2}}{1+\gamma_{1}{ }^{2}+\gamma_{2}{ }^{2}}\right)^{1 / 2} .
$$


This latter formula is recommended for numerical quadrature together with (6) for arguments of $F$ close to 1. Details are given under Computational Considerations.

Series Representations. The integral representation (3) provides a starting point for several series. We use first the formula [5, p. 7]

$$
e^{z \cos \theta}=\sum_{n=0}^{\infty} \epsilon_{n} I_{n}(z) \cos n \theta, \quad \begin{aligned}
\epsilon_{n} & =1, n=0 \\
& =2, n \neq 0
\end{aligned}
$$

on the last integral of (3) where $I_{n}$ is the modified Bessel function of the first kind. This produces the Laplace transform of $I_{n}$ which we evaluate by means of $[6, p .197$ ]

$$
\int_{0}^{\infty} e^{-p t} t^{\mu-1 / 2} I_{2 \nu}\left(2 \alpha t^{1 / 2}\right) d t=\frac{\Gamma(\mu+\nu+1 / 2)}{\Gamma(2 \nu+1)} \frac{\alpha^{2 \nu}}{p^{\mu+\nu+1 / 2}} \Phi\left(\mu+\nu+\frac{1}{2}, 2 \nu+1, \frac{\alpha^{2}}{p}\right)
$$

where $\Phi$ is the confluent hypergeometric function. Then, with $A=\left(\gamma_{1}^{2}+\gamma_{2}^{2}\right)^{1 / 2}$ we have,

$$
\begin{aligned}
P= & \frac{1}{2 \pi \Gamma(k / 2)} \sum_{n=0}^{\infty} \frac{(-1)^{n}}{n} \epsilon_{n} \frac{\Gamma(1+n / 2)}{\Gamma(1+n)} \\
& \times A^{n} \int_{0}^{\infty} u^{-1+(k+n) / 2} \exp \left[-\left(1+\gamma_{1}{ }^{2}+\gamma_{2}{ }^{2}\right) u\right] \Phi\left(\frac{n}{2}+1, n+1, A^{2} u\right) d u \\
& \times \int_{\theta_{1}}^{\theta_{2}} \cos n(\theta-\varphi) d \theta
\end{aligned}
$$

and with (13) and (14),

$$
\begin{aligned}
P= & \frac{1}{2 \pi^{1 / 2} \Gamma(k / 2)} \sum_{n=0}^{\infty} \frac{(-1)^{n} \delta_{n} S_{n}\left(\theta_{1}, \theta_{2}\right)}{\left(1+\gamma_{1}^{2}+\gamma_{2}^{2}\right)^{k / 2}} \frac{\Gamma\left(\frac{k+n}{2}\right)}{\Gamma\left(\frac{n+1}{2}\right)}\left(\frac{c}{2}\right)^{n} \\
& \times F\left(\frac{n}{2}+1, \frac{n+k}{2}, n+1, c^{2}\right)
\end{aligned}
$$

where

$$
\begin{aligned}
c & =\left(\frac{{\gamma_{1}}^{2}+\gamma_{2}{ }^{2}}{1+\gamma_{1}{ }^{2}+\gamma_{2}{ }^{2}}\right)^{1 / 2} & & \\
S_{n}\left(\theta_{1}, \theta_{2}\right) & =\theta_{2}-\theta_{1}, & & n=0 \\
& =2 \sin \frac{n\left(\theta_{2}-\theta_{1}\right)}{2} \cos \frac{n\left(\theta_{1}+\theta_{2}-2 \varphi\right)}{2}, & & n \neq 0 .
\end{aligned}
$$

One obtains this same result if the integrals in (3) are exchanged. In the next section under numerical considerations, this formula is recommended together with methods for computing $F\left(n / 2+1,(n+k) / 2, n+1, c^{2}\right)$. 
A simple series expansion of $\exp \left[-2(u v)^{1 / 2} K(\theta)\right]$ in (3) leads to the representation,

$$
P=\frac{1}{2 \pi^{1 / 2} \Gamma(k / 2)} \sum_{n=0}^{\infty} \frac{(-c)^{n}}{\left(1+\gamma_{1}{ }^{2}+\gamma_{2}{ }^{2}\right)^{k / 2}} \frac{\Gamma\left(\frac{k+n}{2}\right)}{\Gamma\left(\frac{n+1}{2}\right)} \int_{\theta_{1}}^{\theta_{2}} \cos ^{n}(\theta-\varphi) d \theta
$$

This integral in $\theta$ can be expressed in terms of incomplete beta functions which are extensively tabulated.

A more exotic formulation is obtained if the Sonine product [5, p. 98]

$$
z^{\nu} e^{-\gamma z}=2^{\nu} \Gamma(\nu) \sum_{n=0}^{\infty}(-1)^{n}(n+\nu) C_{n}{ }^{\nu}(\gamma) I_{\nu+n}(z)
$$

is used in (3). Here again the factor in $\theta$ is separated and we obtain using (13) and (14),

$$
\begin{aligned}
P= & \frac{\Gamma\left(\frac{k+1}{2}\right)}{\pi(k-1)(k-2)} \sum_{n=0}^{\infty} \frac{(-1)^{n}(n+k-2) G_{n}\left(\theta_{1}, \theta_{2}\right)}{\left(1+\gamma_{1}{ }^{2}+\gamma_{2}{ }^{2}\right)^{k / 2}} \frac{\Gamma\left(\frac{n}{2}+1\right)}{\Gamma\left(\frac{n+k-1}{2}\right)}\left(\frac{c}{2}\right)^{n} \\
& \times F\left(\frac{n+k}{2}, \frac{n}{2}+1 ; n+k-1 ; c^{2}\right)
\end{aligned}
$$

where $G_{n}\left(\theta_{1}, \theta_{2}\right)$ is an integral of the Gegenbauer polynomial $C_{n}{ }^{(k-2)}(\cos (\theta-\varphi))$

$$
G_{n}\left(\theta_{1}, \theta_{2}\right)=\int_{\theta_{1}-\varphi}^{\theta_{2}-\varphi} C_{n}{ }^{(k-2)}(\cos \theta) d \theta .
$$

But

$$
C_{n}{ }^{\nu}(\cos \theta)=\sum_{m=0}^{n} \frac{(\nu)_{m}(\nu)_{n-m}}{m !(n-m) !} \cos (n-2 m) \theta
$$

and

$$
G_{n}\left(\theta_{1}, \theta_{2}\right)=\sum_{m=0}^{n} \frac{(k-2)_{m}(k-2)_{n-m}}{m !(n-m) !} S_{n, m}\left(\theta_{1}, \theta_{2}\right)
$$

where

$$
\begin{array}{rlrl}
S_{n, m}\left(\theta_{1}, \theta_{2}\right) & =\theta_{2}-\theta_{1}, & n=2 m \\
& \frac{2 \sin \frac{(n-2 m)}{2}\left(\theta_{2}-\theta_{1}\right) \cos \frac{(n-2 m)}{2}\left(\theta_{1}+\theta_{2}-2 \varphi\right)}{n-2 m}, & & n \neq 2 m
\end{array}
$$

and $(a)_{m}=a(a+1) \cdots(a+m-1)$.

The case for $\rho=0, k=1$ can be integrated in closed form

$$
P=\frac{1}{2 \pi}\left\{\tan ^{-1}\left(\frac{t_{1} t_{2}}{\sqrt{1+t_{1}{ }^{2}+t_{2}^{2}}}\right)+\tan ^{-1} t_{1}+\tan ^{-1} t_{2}+\frac{\pi}{2}\right\}
$$


while for $k=1$ and any $\rho$, series (10) with the aid of (12) can be written as the solution of a potential problem in the unit circle

$$
\begin{aligned}
P & =\frac{1}{2 \pi} \int_{\theta_{1}-\pi}^{\theta_{2-\pi}} \frac{\left(1-r^{2}\right) d \theta}{1+r^{2}-2 r \cos (\theta-\varphi)} \\
& =\frac{1}{\pi} \tan ^{-1} \frac{2 v}{u^{2}+v^{2}-1}+\left\{\begin{array}{l}
0 \\
1
\end{array}\right\} \quad \begin{array}{l}
u^{2}+v^{2} \geqq 1 \\
u^{2}+v^{2}<1
\end{array}
\end{aligned}
$$

where $-\pi / 2 \leqq \tan ^{-1} t \leqq \pi / 2$ and

$$
\begin{array}{ll}
u=\frac{2 r \sin \varphi}{A\left(1+r^{2}+2 r \cos \varphi\right)}, & v=\frac{1-r^{2}}{A\left(1+r^{2}+2 r \cos \varphi\right)} \\
r=\frac{\sqrt{\gamma_{1}^{2}+\gamma_{2}{ }^{2}}}{1+\sqrt{1+\gamma_{1}{ }^{2}+\gamma_{2}{ }^{2}}}, & A=\tan \left(\left(\theta_{2}-\pi\right) / 2\right) .
\end{array}
$$

The fact that $\theta_{1}-\pi=-\left(\theta_{2}-\pi\right)$ has been used together with the conformal map of the unit circle into the upper half $w$-plane

$$
w=\frac{i}{A} \frac{(1-z)}{(1+z)}, \quad|z| \leqq 1
$$

with $z=\exp \left[i\left(\theta_{2}-\pi\right)\right] \rightarrow w=1, z=\exp \left[-i\left(\theta_{2}-\pi\right)\right] \rightarrow w=-1$.

Computational Considerations. For numerical evaluation, attention was directed toward (9) and (10), although (11) appears to be a possibility in view of Gautschi's [7] results. The quadrature in (9) (together with (6)) presented no problems in a Romberg integration routine, and series (10) reproduced the quadrature results (except for occasional discrepancies in the fifth digit) in comparable computer time for $k \geqq 2$ with a maximum of 350 terms. Actually fewer terms (50 to 100) were required for the larger $k$ values since the variance of the distribution decreases with $k$ (approaching that of a normal distribution) giving smaller $c$ values for a given percent point. For $k=1$, the series required more than 400 terms in some cases for just three-decimal place agreement; while five-decimal place agreement between the quadrature and series was the norm for $k \geqq 2$ on relative and absolute error tests of $5 \times 10^{-5}$ respectively. The quadrature mesh size was halved while the upper index of the truncated series was incremented by 50 terms until the respective tests were met.

Altogether, 1335 comparisons were made on parameter values

$$
\rho=-.9,-.5,0, .5, .9 \quad k=1,2,5,10,25,50
$$

and $\left(t_{1}, t_{2}\right)$ pairs on circles about the origin at $45^{\circ}$ angular spacing beginning at $45^{\circ}$ and ending at $225^{\circ}$ since there is symmetry about the line $t_{1}=t_{2}$. The increment in the radii varied with $k$ to cover the most significant portion of each distribution. The computation in the $\left(t_{1}, t_{2}\right)$ plane was terminated for a given $\rho$ and $k$ on the condition $P \geqq .99$ on the $45^{\circ}$ ray. The computations were done in single-precision arithmetic (approximately $10 \frac{1}{2}$ digits) on a CDC 3600 computer. A relative error test of $5 \times 10^{-7}$ was used for the $\bar{w}$ functions described below. 
The computation of $F(1, k / 2,(k+3) / 2, x)$ for (9) is rapid with the series expansions about 0 and 1 since $x$ or $1-x$ can always be taken less than $1 / 2$. The continued fraction $[4$, p. 88$]$

$$
\begin{aligned}
R_{n} & =1-\frac{u_{n} x}{1-v_{n} x / R_{n+1}}, \\
u_{n} & =\frac{(b+n-1)(c+n-2)}{(c+2 n-3)(c+2 n-2)}, \\
v_{n} & =\frac{n(c-b+n-1)}{(c+2 n-2)(c+2 n-1)}, \\
F(1, b ; c ; x) & =1 / R_{1}
\end{aligned}
$$

also shows promise in numerical evaluation. Notice that the continued fraction may be used on the right side of (6) also.

The computation of $F\left((n+k) / 2, n / 2+1 ; n+1 ; c^{2}\right)$ produced some difficulties. For large $k$, the series representations about 0 and 1 do not converge rapidly enough in the middle of the interval to be useful. Furthermore, the parameters for even $k$ put the $F$ function in the exceptional (logarithmic) case and makes evaluation more difficult about 1 . The following recursion methods proposed by Gautschi [7] were used with success. Note that the contiguous relation

$$
\begin{aligned}
& (c-a) F(a-1, b ; c ; z)+(2 a-c-a z+b z) F(a, b ; c ; z) \\
& \quad=a(1-z) F(a+1, b ; c ; z)
\end{aligned}
$$

produces a recurrence relation in the parameter $a$.

Case $1 . k$ odd, $k \geqq 1$.

The contiguous relation above, under the parameterization

$$
a=(n+1) / 2+m, \quad b=n / 2+1, \quad c=n+1
$$

reduces to

$$
\begin{aligned}
y_{m+1} & =\frac{1}{1-z}\left[\frac{n+1-2 m}{n+1+2 m} y_{m-1}+\frac{4 m+(1-2 m) z}{(n+1+2 m)} y_{m}\right], m=1,2, \cdots, \frac{k-3}{2} \\
y_{m} & =F\left(\frac{n+1}{2}+m, \frac{n}{2}+1 ; n+1 ; z\right), m=0,1,2, \cdots, \frac{k-1}{2} .
\end{aligned}
$$

It is clear that the difference equation with the initial values

$y_{0}=F((n+1) / 2, n / 2+1 ; n+1 ; z), \quad y_{1}=F((n+3) / 2, n / 2+1 ; n+1 ; z)$

generates a "dominant" solution so that forward recurrence works. Now, $y_{0}$ and $y_{1}$ can be identified in terms of simple algebraic quantities

$$
\begin{aligned}
y_{0}=\frac{2^{n}}{(1-z)^{1 / 2}\left(1+(1-z)^{1 / 2}\right)^{n}}, \quad y_{1}=\frac{2^{n}\left(1-n(1-z)^{1 / 2}\right)}{(n+1)(1-z)^{3 / 2}\left(1+(1-z)^{1 / 2}\right)^{n}}, \\
n \geqq 1
\end{aligned}
$$

by means of the relations 


$$
\begin{aligned}
F\left(a, a+\frac{1}{2} ; 2 a ; z\right) & =\frac{1}{(1-z)^{1 / 2}}\left(\frac{1+(1-z)^{1 / 2}}{2}\right)^{1-2 a}, \\
F\left(\frac{n+1}{2}+1, \frac{n}{2}+1 ; n+1 ; z\right) & =\frac{4}{n+1} \frac{d}{d z} F\left(\frac{n}{2}, \frac{n+1}{2} ; n ; z\right) .
\end{aligned}
$$

For $n=0$, and all $k \geqq 1$

$$
F(1, k / 2 ; 1 ; z)=(1-z)^{-k / 2} .
$$

Case 2 . $k$ even, $k \geqq 2$.

The contiguous relation under the parameterization

$$
a=n / 2+m, \quad b=n / 2+1, \quad c=n+1
$$

gives

$$
\begin{array}{r}
y_{m+1}=\frac{1}{1-z}\left[\frac{n+2-2 m}{n+2 m} y_{m-1}+2 \frac{[(2 m-1)-(m-1) z]}{n+2 m} y_{m}\right] \\
m=1,2, \cdots, \frac{k-2}{2}
\end{array}
$$

with

$$
\begin{aligned}
& y_{m}=F(n / 2+m, n / 2+1, n+1 ; z), \quad m=0,1, \cdots, k / 2 \\
& y_{0}=F(n / 2, n / 2+1 ; n+1 ; z), \quad y_{1}=F(n / 2+1, n / 2+1 ; n+1 ; z) .
\end{aligned}
$$

Here again forward recursion works, but the computation of $y_{0}$ and $y_{1}$ is not as simple as in Case 1. The relations

$$
\begin{aligned}
y_{0} & =\frac{-4}{n(1-z)^{-1+n / 2}} \frac{d}{d z}\left[(1-z)^{n / 2} F\left(\frac{n}{2}, \frac{n}{2} ; n ; z\right)\right], \\
y_{1} & =\frac{4}{n} \frac{d}{d z} F\left(\frac{n}{2}, \frac{n}{2} ; n ; z\right), \\
Q_{\nu}(z) & =\frac{\pi^{1 / 2} \Gamma(\nu+1)}{2^{\nu+1} \Gamma(\nu+3 / 2)} \frac{1}{(1+z)^{\nu+1}} F\left(\nu+1, \nu+1 ; 2+2 \nu ; \frac{2}{1+z}\right),|1+z|>2
\end{aligned}
$$

provide the identification in terms of Legendre functions of the second kind:

$$
Q_{-1+n / 2}\left(\frac{2-z}{z}\right)=\frac{\pi^{1 / 2} \Gamma\left(\frac{n}{2}\right)}{2^{n / 2} \Gamma\left(\frac{n+1}{2}\right)}\left(\frac{z}{2}\right)^{n / 2} F\left(\frac{n}{2}, \frac{n}{2} ; n ; z\right) .
$$

Differentiation together with

$$
Q_{\nu}{ }^{\prime}(x)=\frac{\nu+1}{1-x^{2}}\left[x Q_{\nu}(x)-Q_{\nu+1}(x)\right]
$$

produces 


$$
\begin{aligned}
x & =(2-z) / z \\
y_{0} & =\frac{2^{n} \Gamma\left(\frac{n+1}{2}\right)}{\pi^{1 / 2} \Gamma\left(\frac{n}{2}\right) z^{n / 2}}\left[Q_{-1+n / 2}(x)+Q_{n / 2}(x)\right] \\
y_{1} & =\frac{1}{1-z} \frac{2^{n} \Gamma\left(\frac{n+1}{2}\right)}{\pi^{1 / 2} \Gamma\left(\frac{n}{2}\right) z^{n / 2}}\left[Q_{-1+n / 2}(x)-Q_{n / 2}(x)\right] .
\end{aligned}
$$

Thus, for $n$ odd, $n \geqq 1$, we must generate the half-odd Legendre functions of the second kind starting with

$$
Q_{-1 / 2}(x)=\left(\frac{2}{x+1}\right)^{1 / 2} K\left(\left(\frac{2}{x+1}\right)^{1 / 2}\right)
$$

where $K$ is the complete elliptic integral of the first kind. The procedure of Gautschi [7] is very efficient since all half-odd functions can be produced to a specified relative error by means of the algorithm:

$$
\begin{aligned}
& r_{\nu}{ }^{(\nu)}=\frac{2 \nu+1}{2(\nu+1)} \cdot \frac{1}{x+\left(x^{2}-1\right)^{1 / 2}}, \quad r_{m-1}^{(\nu)}=\frac{-b_{m}}{a_{m}+r_{m}{ }^{(\nu)}}, \quad m=\nu, \nu-1, \cdots, 1 \\
& s_{\nu}{ }^{(\nu)}=0, s_{m-1}^{(\nu)} \quad=r_{m-1}^{(\nu)}\left(\lambda_{m}+s_{m}{ }^{(\nu)}\right) \\
& w_{0}{ }^{(\nu)}=\frac{s}{\lambda_{0}+s_{0}{ }^{(\nu)}}, w_{m}{ }^{(\nu)} \quad=r_{m-1}^{(\nu)} w_{m-1}^{(\nu)}, \quad m=1,2, \cdots, M \\
& a_{m}=\frac{-4 m x}{2 m+1}, b_{m} \quad=\frac{2 m-1}{2 m+1}, \quad m=1,2, \cdots, \nu \\
& s=\frac{\pi / 2^{1 / 2}}{(x-1)^{1 / 2}}, \lambda_{0} \quad=1, \lambda_{m}=2, \quad m=1,2, \cdots, \nu
\end{aligned}
$$

with

$$
w_{m}=Q_{-1 / 2+m}(x)=\lim _{\nu \rightarrow \infty} w_{m}^{(\nu)}, \quad m=0,1,2, \cdots, M .
$$

Here, at least two applications of the procedure are needed for different values of $\nu: \nu_{2}>\nu_{1}>$ largest index of interest $=M$. The results for the indices of interest are tested for relative error and the procedure for $\nu_{3}>\nu_{2}$ is reapplied if the two sets of numbers do not compare favorably. Here,

$$
r_{m}=\frac{Q_{m+1 / 2}(x)}{Q_{m-1 / 2}(x)}=\lim _{\nu \rightarrow \infty} r_{m}{ }^{(\nu)}
$$

and the asymptotic form for large $\nu$ was used for $Q_{\nu}(x)$ to start the recursion with $m=\nu$. For $n$ even, $n \geqq 2$, the computational procedure is the same as for odd $n$ except for the replacements 


$$
\begin{aligned}
w_{0}{ }^{(\nu)} & =Q_{0}(x)=\frac{1}{2} \ln \left(\frac{x+1}{x-1}\right), \quad w_{m}=Q_{m}(x)=\lim _{\nu \rightarrow \infty} w_{m}{ }^{(\nu)} \\
a_{m} & =-\frac{2 m+1}{m+1} x, \quad b_{m}=\frac{m}{m+1}, \quad m=1,2, \cdots . \\
r_{\nu}{ }^{(\nu)} & =\frac{2(\nu+1)}{(2 \nu+3)} \frac{1}{x+\left(x^{2}-1\right)^{1 / 2}}
\end{aligned}
$$

and the fact that the $s$ sequence need not be generated because of the explicit form for $w_{0}$. As explained in the reference, the $Q$ 's are "minimal" solutions of a three-term recurrence relation

$$
w_{m+1}+a_{m} w_{m}+b_{m} w_{m-1}=0, \quad m=1,2, \cdots .
$$

The algorithm for these solutions constitutes backward recurrence on the ratios followed by forward recurrence and normalization. In this analysis normalization (finding the constant of proportionality) is achieved by summing the series

$$
s=\lambda_{0} Q_{-1 / 2}(x)+\sum_{m=1}^{\infty} \lambda_{m} Q_{-1 / 2+m}(x)
$$

in the first case and specifying $Q_{0}(x)$ in the second case. For $n$ fixed and moderately large (50-100), $Q_{n / 2}(x)$ decreases rapidly as $x$ increases past 5 . Better scaling results for both large and small $x$ by modification of the algorithm to incorporate $z^{-n / 2}$ as a multiplying factor of $Q_{n / 2}(x)$. The new algorithm for odd and even $n$ is

$$
\begin{aligned}
\bar{w}_{m} & =[(x+1) / 2]^{-1 / 2+m} Q_{-1 / 2+m}(x), \quad \bar{w}_{m}=[(x+1) / 2]^{m} Q_{m}(x), \quad m=0,1,2, \cdots \\
\bar{r}_{\nu}{ }^{(\nu)} & =\frac{(x+1)}{2} r_{v}{ }^{(\nu)}, \quad \bar{r}_{m-1}^{(\nu)}=\frac{-\bar{b}_{m}}{\bar{a}_{m}+\bar{r}_{m}{ }^{(\nu)}}, \\
\bar{a}_{m} & =\frac{(x+1)}{2} a_{m}, \bar{b}_{m}=\frac{(x+1)^{2}}{4} b_{m}, \\
\bar{w}_{m}{ }^{(\nu)} & =\bar{r}_{m-1}^{(\nu)} \bar{w}_{m-1}^{(\nu)}, \quad m=1,2, \cdots, M
\end{aligned}
$$

with normalization achieved by computing

$$
\bar{w}_{0}=\frac{Q_{-1 / 2}(x)}{((x+1) / 2)^{1 / 2}} \text { and } \bar{w}_{0}=Q_{0}(x) .
$$

$Q_{-1 / 2}(x)$ is obtained from the original algorithm. The expressions for $y_{0}$ and $y_{1}$ become

$$
\begin{aligned}
y_{0}= & \frac{2^{n} \Gamma\left(\frac{n+1}{2}\right)}{\pi^{1 / 2} \Gamma\left(\frac{n}{2}\right)}\left[\frac{(x+1)}{2} \bar{w}_{m-1}(x)+\bar{w}_{m}(x)\right] \\
y_{1}= & \frac{2^{n} \Gamma\left(\frac{n+1}{2}\right)}{(1-z) \pi^{1 / 2} \Gamma\left(\frac{n}{2}\right)}\left[\left(\frac{x+1}{2}\right) \bar{w}_{m-1}(x)-\bar{w}_{m}(x)\right]
\end{aligned}
$$


for each sequence corresponding to even and odd $n$.

Appendix. We wish to evaluate

$$
I=\int_{0}^{\infty} e^{-z t} t^{-1+\beta / 2} D_{-\nu}\left( \pm 2(k t)^{1 / 2}\right) d t
$$

for $z>k \geqq 0, \beta>0$. We start with

$$
\begin{aligned}
D_{-\nu}(z)=2^{-\nu / 2} e^{-z^{2} / 4}\left[\frac{\Gamma\left(\frac{1}{2}\right)}{\Gamma\left(\frac{1+\nu}{2}\right)} \Phi\left(\frac{\nu}{2}, \frac{1}{2} ; \frac{z^{2}}{2}\right)\right. & \\
& \left.+\frac{z}{\sqrt{ } 2} \frac{\Gamma\left(-\frac{1}{2}\right)}{\Gamma\left(\frac{\nu}{2}\right)} \Phi\left(\frac{1+\nu}{2}, \frac{3}{2} ; \frac{z^{2}}{2}\right)\right] .
\end{aligned}
$$

Then,

$$
\begin{aligned}
I= & \frac{2^{-\nu / 2} \Gamma\left(\frac{1}{2}\right)}{\Gamma\left(\frac{1+\nu}{2}\right)} \int_{0}^{\infty} e^{-(z+k) t} t^{-1+\beta / 2} \Phi\left(\frac{\nu}{2}, \frac{1}{2} ; 2 k t\right) d t \\
& \pm 2^{(1-\nu) / 2} \frac{\sqrt{ } k \Gamma\left(-\frac{1}{2}\right)}{\Gamma\left(\frac{\nu}{2}\right)} \int_{0}^{\infty} e^{-(z+k) t} t^{-1+(\beta+1) / 2} \Phi\left(\frac{1+\nu}{2}, \frac{3}{2} ; 2 k t\right) d t
\end{aligned}
$$

for $z>k>0$ since $\Phi(a, c ; x) \sim \Gamma(c) / \Gamma(a) e^{x} x^{a-c}$ for $x \rightarrow \infty$. The Laplace transform

$$
\int_{0}^{\infty} e^{-p t} t^{b-1} \Phi(a, c ; \gamma t) d t=\frac{\Gamma(b)}{p^{b}} F\left(a, b ; c ; \frac{\gamma}{p}\right), \quad|p|>|\gamma|
$$

converts the right side to

$$
\begin{aligned}
I=\frac{2^{-\nu / 2}}{(z+k)^{\beta / 2}}\left[\frac{\Gamma\left(\frac{1}{2}\right) \Gamma\left(\frac{\beta}{2}\right)}{\Gamma\left(\frac{1+\nu}{2}\right)} F\left(\frac{\nu}{2}, \frac{\beta}{2} ; \frac{1}{2} ; \frac{2 k}{z+k}\right)\right. \\
\left.\qquad\left(\frac{2 k}{z+k}\right)^{1 / 2} \frac{\Gamma\left(-\frac{1}{2}\right) \Gamma\left(\frac{\beta+1}{2}\right)}{\Gamma\left(\frac{\nu}{2}\right)} F\left(\frac{1+\nu}{2}, \frac{\beta+1}{2} ; \frac{3}{2} ; \frac{2 k}{z+k}\right)\right]
\end{aligned}
$$

and with the Legendre formula

$$
\Gamma(2 z)=\frac{2^{2 z-1}}{\pi^{1 / 2}} \Gamma(z) \Gamma(z+1 / 2)
$$

we have 


$$
\begin{aligned}
I=\frac{2^{1-\beta-\nu / 2} \pi^{1 / 2}}{(z+k)^{\beta / 2}} \frac{\Gamma(\beta)}{\Gamma\left(\frac{\nu+\beta+1}{2}\right)}\left[\frac{\Gamma\left(\frac{1}{2}\right) \Gamma\left(\frac{\nu+\beta+1}{2}\right)}{\Gamma\left(\frac{1+\nu}{2}\right) \Gamma\left(\frac{\beta+1}{2}\right)} F\left(\frac{\nu}{2}, \frac{\beta}{2} ; \frac{1}{2} ; \frac{2 k}{z+k}\right)\right. \\
\pm\left(\frac{2 k}{z+k}\right)^{1 / 2} \frac{\Gamma\left(-\frac{1}{2}\right) \Gamma\left(\frac{\nu+\beta+1}{2}\right)}{\Gamma\left(\frac{\beta}{2}\right) \Gamma\left(\frac{\nu}{2}\right)} \\
\left.\times F\left(\frac{1+\nu}{2}, \frac{\beta+1}{2} ; \frac{3}{2} ; \frac{2 k}{z+k}\right)\right] .
\end{aligned}
$$

For the positive sign, this is the right side of an analytic continuation formula for $F(\nu / 2, \beta / 2,(\nu+\beta+1) / 2,(z-k) /(z+k))$. Therefore, one gets the standard result found in most tables,

$$
\begin{aligned}
\int_{0}^{\infty} e^{-z t} t^{-1+\beta / 2} D_{-\nu}\left(2(k t)^{1 / 2}\right) d t & =\frac{\pi^{1 / 2} 2^{1-\beta-\nu / 2}}{(z+k)^{\beta / 2}} \\
& \times \frac{\Gamma(\beta)}{\Gamma\left(\frac{\nu+\beta+1}{2}\right)} F\left(\frac{\nu}{2}, \frac{\beta}{2} ; \frac{\nu+\beta+1}{2} ; \frac{z-k}{z+k}\right) .
\end{aligned}
$$

There is, however, a more coherent way of presenting both results in terms of associated Legendre functions, by means of the relation

$$
\begin{aligned}
\frac{\left(1-x^{2}\right)^{\bar{\mu} / 2} 2^{-\bar{\mu}}}{\pi^{1 / 2}} P_{\bar{\nu}}^{\bar{\mu}}( \pm x)= & \frac{F\left(-\frac{\bar{\mu}+\bar{\nu}}{2}, \frac{1+\bar{\nu}-\bar{\mu}}{2} ; \frac{1}{2} ; x^{2}\right)}{\Gamma\left(\frac{1-\bar{\nu}-\bar{\mu}}{2}\right) \Gamma\left(1+\frac{\bar{\nu}-\bar{\mu}}{2}\right)} \\
& \mp \frac{2 x F\left(\frac{1-\bar{\nu}-\bar{\mu}}{2}, 1+\frac{\bar{\nu}-\bar{\mu}}{2} ; \frac{3}{2} ; x^{2}\right)}{\Gamma\left(\frac{1+\bar{\nu}-\bar{\mu}}{2}\right) \Gamma\left(-\frac{\bar{\mu}+\bar{\nu}}{2}\right)}, \quad-1<x<1
\end{aligned}
$$

if we let $\bar{\nu}=(\beta-\nu-1) / 2$ and $\bar{\mu}=(1-\nu-\beta) / 2$.

Then,

$$
I=\frac{\pi^{1 / 2} 2^{(1-\beta) / 2} \Gamma(\beta)}{(z+k)^{\beta / 2}}\left(1-x^{2}\right)^{\bar{\mu} / 2} P_{\bar{\nu}}^{\bar{\mu}}( \pm x)
$$

where

$$
x=\left(\frac{2 k}{z+k}\right)^{1 / 2}, \quad z>k
$$

We also have 


$$
P_{\bar{\nu}}^{\bar{\mu}}(x)=\frac{1}{\Gamma(1-\bar{\mu})}\left(\frac{1+x}{1-x}\right)^{\bar{\mu} / 2} F\left(-\bar{\nu}, \bar{\nu}+1 ; 1-\bar{\mu} ; \frac{1-x}{2}\right), \quad-1<x<1
$$

so that

$$
\begin{aligned}
I= & \frac{\pi^{1 / 2} 2^{(1-\beta) / 2} \Gamma(\beta)}{(z+k)^{\beta / 2} \Gamma\left(\frac{1+\nu+\beta}{2}\right)}(1 \pm x)^{(1-\nu-\beta) / 2} \\
& \quad \times F\left(\frac{1+\nu-\beta}{2}, \frac{1+\beta-\nu}{2} ; \frac{1+\nu+\beta}{2} ; \frac{1 \mp x}{2}\right) .
\end{aligned}
$$

Notice that a quadratic transformation [4, p. 112]

$$
\begin{aligned}
& F(a, 1-a ; c ; z)=(1-z)^{c-1} F\left(\frac{c-a}{2}, \frac{c+a-1}{2} ; c ; 4 z(1-z)\right) \\
& \operatorname{Re} z<1 / 2,|4 z(1-z)|<1
\end{aligned}
$$

applies for the negative sign in the argument of $F$ giving formula (15). On the other hand this quadratic transformation does not apply to the other sign since the argument is greater than $1 / 2$.

Sandia Laboratory

Albuquerque, New Mexico 87110

University of Kansas

Lawrence, Kansas 66044

1. C. W. Dunnett, "A multiple comparison procedure for comparing several treatments with a control," J. Amer. Statist. Assoc., v. 50, 1955, pp. 1096-1121.

2. C. W. DunNetT \& M. Sobel, "A bivariate generalization of Student's $t$-distribution, with tables for certain special cases," Biometrika, v. 41, 1954, pp. 153-169. MR 15, 885.

3. C. W. Dunnett \& M. Sobel, "Approximation to the probability integral and certain percentage points of a multivariate analogue of Student's $t$-distribution," Biometrika, v. 42, 1955, pp. 258-260. MR 16, 840 .

4. A. ERdÉLYI, et al., Higher Transcendental Functions, Vol. 1, McGraw-Hill, New York, 1953. MR 15, 419.

5. A. ERDÉLYI, et al., Higher Transcendental Functions, Vol. 2, McGraw-Hill, New York, 1953. MR 15, 419.

6. A. ERDÉLYI, et al., Tables of Integral Transforms, Vol. 1, McGraw-Hill, New York, 1954. MR 15, 868.

7. W. GAUTSCHI, "Computational aspects of three-term recurrence relations," SIAM Rev., v. 9, 1967, pp. 24-82. MR 35 \#3927.

8. S. S. GUPTA \& M. SOBFL, "On a statistic which arises in selection and ranking problems," Ann. Math. Statist., v. 28, 1957, pp. 957-967. MR 20 \#366.

9. S. S. GUPTA, "Probability integrals of multivariate normal and multivariate $t$," Ann. Math. Statist., v. 34, 1963, pp. 792-828. MR 27 \#2048.

10. S. JoHN, "On the evaluation of the probability integral of the multivariate $t$-distribution," Biometrika, v. 48, 1961, pp. 409-417. MR 26 \#1951.

11. S. JoHN, "Methods for the evaluation of probabilities of polygonal and angular regions when the distribution is bivariate $t$," Sankhyā, Ser. A, v. 26, 1964, pp. 47-54. MR 33 \#6736.

12. P. R. Krishnatah \& J. V. Armitage, Percentage Points of the Multivariate t-Distribution, ARL 65-199, Aerospace Research Laboratories, Wright-Patterson Air Force Base, Ohio, 1965.

13. N. N. Lebedev, Special Functions and Their Applications, Fizmatgiz, Moscow, 1963; English transl., Prentice-Hall, Englewood Cliffs, N. J., 1965. MR 30 \#4987; MR 30 \#4988.

14. D. B. OwEN, "A special case of a bivariate non-central $t$-distribution," Biometrika, v. 52 , 1965, pp. 437-447. MR 34 \#5190.

15. K. C. S. Pillai \& K. V. Ramachandran, "On the distribution of the ratio of the $i$ th observation in an ordered sample from a normal population to an independent estimate of the standard deviation," Ann. Math. Statist., v. 25, 1954, pp. 565-572. MR 16, 270. 